\title{
Posterior reversible encephalopathy syndrome secondary to dengue fever: a case report
}

\author{
SW Cheo' ${ }^{*}$, MRCP (UK), HJ Wong ${ }^{2}$, MRCP (UK), EK Ng ${ }^{3}$, MRCP (UK), QJ Low ${ }^{4}, \operatorname{MRCP}(\mathrm{UK})$, YK Chia ${ }^{5}$, MRCP (UK)
}

\author{
${ }^{1}$ Department of Internal Medicine, Hospital Lahad Datu, Lahad Datu, Sabah, Malaysia \\ ${ }^{2}$ Department of Internal Medicine, Hospital Duchess of Kent, Sandakan, Sabah, Malaysia \\ ${ }^{3}$ Department of Internal Medicine, Hospital Tawau, Sabah, Malaysia \\ ${ }^{4}$ Department of Internal Medicine, Hospital Sultanah Nora Ismail, Batu Pahat, Johor, Malaysia \\ ${ }^{5}$ Department of Internal Medicine, Hospital Queen Elizabeth, Kota Kinabalu, Sabah, Malaysia
}

* Corresponding author: cheosengwee@gmail.com

Hong Kong Med J 2021;27:55-7

https://doi.org/10.12809/hkmj208509

\section{Case report}

A 15-year-old boy with good past health presented with a 1-day history of fever, four episodes of vomiting, and lethargy. He denied diarrhoea, headache, or any neurological symptoms. On arrival, he had blood pressure 106/36 $\mathrm{mm} \mathrm{Hg}$, heart rate $127 \mathrm{bpm}$, temperature $39^{\circ} \mathrm{C}$, and oxygen saturation $99 \%$ on air. He had reduced pulse volume with normal capillary refilling time. Systemic examination was otherwise unremarkable. Full blood count analysis revealed a haemoglobin level of $14.7 \mathrm{~g} / \mathrm{dL}$, white cell count of $9.31 \times 10^{9} / \mathrm{L}$, and platelet count of $240 \times 10^{9} / \mathrm{L}$. The patient's blood test results were positive for dengue non-structural protein 1 , and negative for dengue immunoglobulin $M$ and immunoglobulin G. His renal profile was normal, with mild raised liver enzymes: alanine aminotransferase $82 \mathrm{U} / \mathrm{L}$ and aspartate aminotransferase $310 \mathrm{U} / \mathrm{L}$.

In view of the tachycardia and reduced pulse volume, he was treated as a case of severe dengue fever with compensated shock. He was admitted to the intensive care unit and treated with fluid boluses according to standard protocol. On day 3 of illness he developed severe plasma leakage and was intubated for respiratory distress. His condition soon stabilised and he remained normotensive throughout his stay in intensive care unit. He was extubated on day 7 when he entered a recovery phase. At that time, he had a normal level of consciousness.

Three days later (day 10 of illness), he became encephalopathic, disorientated and able to obey only single step commands. Examination revealed a Glasgow Coma Scale score of E4V4M6 with normal motor power over both upper and lower limbs. A plain computed tomography (CT) brain scan showed hypodensities at bilateral occipital regions and semiovale, predominantly involving the white matter, and suggestive of posterior reversible encephalopathy syndrome (PRES) [Fig 1]. A diagnostic lumbar puncture was offered but refused by his parents. Blood and urine cultures were negative and he was prescribed a 1-week course of

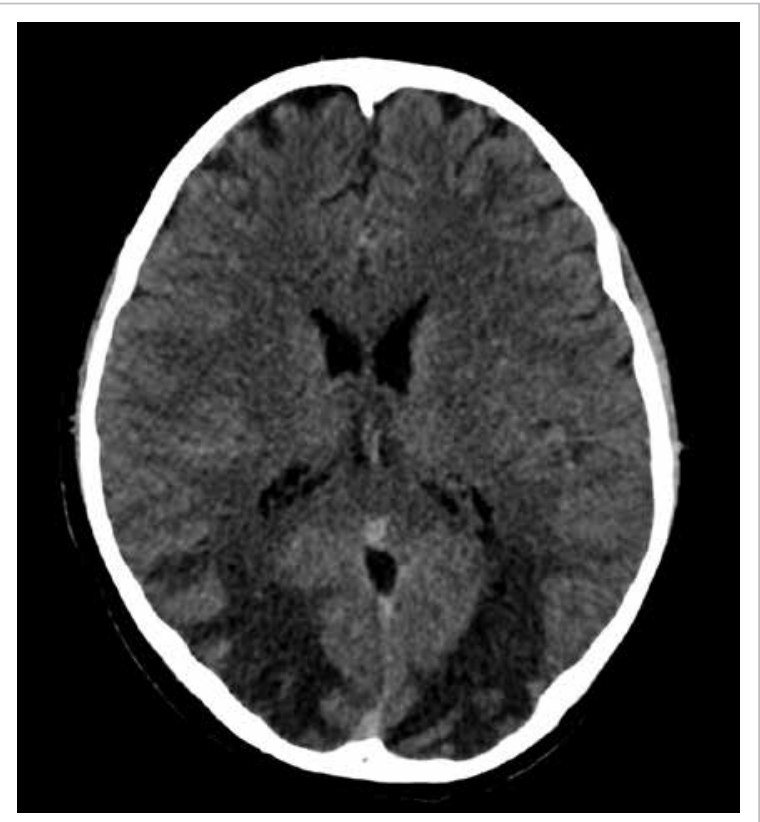

FIG I. Plain computed tomography brain showing symmetrical hypodensities at bilateral occipital regions and semiovale, predominantly involving the white matter, suggestive of posterior reversible encephalopathy syndrome

intravenous meropenem. He subsequently improved and was discharged well on day 16. Serum dengue polymerase chain reaction was later confirmed as DEN-2. At clinic follow-up, the patient was well and asymptomatic. Repeat CT brain at 3 months showed complete resolution of white matter oedema (Fig 2).

\section{Discussion and conclusion}

Dengue fever is an important public health problem in the tropics. At present, it is endemic in more than 100 countries. The World Health Organization estimates there to be around 390 million infections per year with 96 million manifesting clinically. ${ }^{1}$ The clinical features vary from those of a mild febrile 


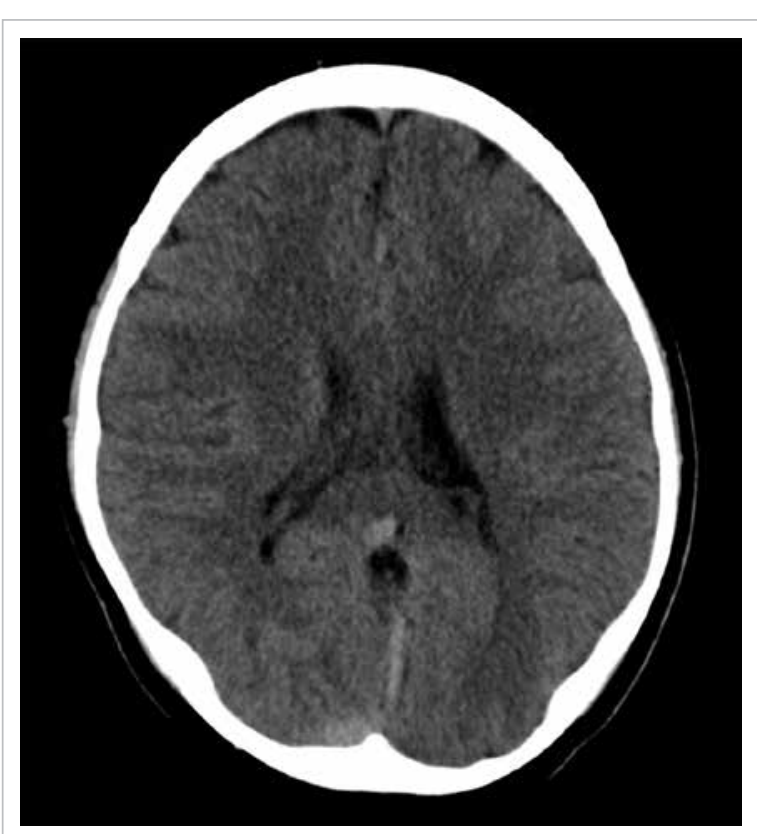

FIG 2. Follow-up computed tomography brain showing complete resolution of hypodensities illness to severe dengue with systemic complications. Systemic complications include shock, bleeding, plasma leakage, myocarditis and, in some patients, neurological complications. The latter include dengue encephalitis, dengue encephalopathy, meningitis, acute disseminated encephalomyelitis, and Guillain-Barré syndrome. ${ }^{2}$ Posterior reversible encephalopathy syndrome can occur but is rare.

Posterior reversible encephalopathy syndrome is a distinct clinicoradiological syndrome characterised by headache, seizures, altered consciousness and visual disturbances with the presence of reversible brain vasogenic oedema. ${ }^{3}$ It can be caused by acute hypertension, hypertensive disease in pregnancy, autoimmune diseases (systemic lupus erythematosus, systemic sclerosis), immunosuppressants, cytotoxic agents, renal failure, and infection. An infectious aetiology such as virus and gram-positive or gram-negative sepsis have all been associated with PRES as has dengue virus. ${ }^{4,5}$

The pathophysiology of PRES in dengue is still not fully understood. It is postulated to be related to endothelial dysfunction ${ }^{4}$ that subsequently leads to vasogenic oedema and decreased cerebral blood flow. In addition, inflammatory cytokines can increase vascular permeability with consequent interstitial brain oedema. ${ }^{3}$ Neuroimaging is essential for diagnosis with characteristics that include vasogenic oedema in the parieto-occipital region of both cerebral hemispheres. The subcortical white matter is always affected. Three primary patterns of magnetic resonance imaging (MRI) brain have been described, namely dominant parieto-occipital pattern, holohemispheric watershed pattern, and superior frontal sulcus pattern. Treatment is mainly supportive with removal of causative factors.

In our patient, diagnosis of PRES was based on clinical and radiological features. He developed acute onset encephalopathy coupled with typical neuroimaging features. Brain CT showed symmetrical hypodensities at bilateral occipital lobes and predominantly involving white matter, suggestive of PRES. He was treated conservatively, and he improved. Follow-up CT brain revealed complete resolution of vasogenic oedema. In terms of aetiology, our patient had no history of hypertension or autoimmune disease or recent cytotoxic agent. In addition, blood pressure remained stable throughout his hospital stay with no decompensated shock. The limitation of our case is the lack of MRI facilities. Compared with CT, MRI better delineates lesions and is preferable if available.

In terms of timing of PRES in relation to dengue fever, PRES has been reported to have occurred on day 5 to 6 of illness in one case, ${ }^{4}$ and within the non-structural protein 1 antigen positive period (first 7 days of illness) in another. ${ }^{5}$ In our case, it occurred on day 10 of illness and was considered compatible with the cases reported previously. A diagnosis of PRES secondary to dengue is one of exclusion, supported by typical imaging features and within a reasonable time frame. Alternative causes of PRES should be actively searched for and excluded in every case.

In conclusion, this case highlights PRES as an important differential diagnosis in a dengue patient with neurological deficits. It is important to differentiate PRES from other clinical syndromes since management is mainly supportive and by controlling the underlying conditions. Neuroimaging plays an important role in establishing the diagnosis in the compatible clinical context. The prognosis of PRES is usually good. However, more studies are needed to further elucidate the underlying pathophysiology of PRES in dengue.

\section{Author contributions}

Concept or design: SW Cheo and QJ Low. Acquisition of data: SW Cheo and HJ Wong. Analysis or interpretation of data: HJ Wong and EK Ng. Drafting of the manuscript: SW Cheo and QJ Low. Critical revision of the manuscript for important intellectual content: All authors.

All authors had full access to the data, contributed to the study, approved the final version for publication, and take responsibility for its accuracy and integrity.

\section{Conflicts of interest}

The authors have disclosed no conflicts of interest. 


\section{Funding/support}

This case report received no specific grant from any funding agency in the public, commercial, or not-for-profit sectors.

\section{Ethics approval}

The patient was treated in accordance with the Declaration of Helsinki. The patient provided written informed consent for all treatments and procedures, and written consent for publication.

\section{References}

1. World Health Organization. Dengue and severe dengue: Fact sheet. Available from: https://www.who.int/news- room/fact-sheets/detail/dengue-and-severe-dengue. Accessed 4 Nov 2019.

2. Murthy JM. Neurological complications of dengue infection. Neurol India 2010;58:581-4.

3. Fugate JE, Rabinstein AA. Posterior reversible encephalopathy syndrome: clinical and radiological manifestations, pathophysiology, and outstanding questions. Lancet Neurol 2015;14:914-25.

4. Mai NT, Phu NH, Nghia HD, et al. Dengue-associated posterior reversible encephalopathy syndrome, Vietnam. Emerg Infect Dis 2018;24:402-4.

5. Sohoni CA. Bilateral symmetrical parieto occipital involvement in dengue infection. Ann Indian Acad Neurol 2015;18:358-9. 